\title{
Corrections
}

\section{Correction: Guo et al., A Sensitive Period for GABAergic Interneurons in the Dentate Gyrus in Modulating Sensorimotor Gating}

In the article "A Sensitive Period for GABAergic Interneurons in the Dentate Gyrus in Modulating Sensorimotor Gating” by Nannan Guo, Kaichi Yoshizaki, Ryuichi Kimura, Fumikazu Suto, Yuchio Yanagawa, and Noriko Osumi, which appeared on pages 6691-6704 of the April 10, 2013 issue, the authors regret an error in Figure 2E, where the bar graph for forced swimming test at PW6 was accidentally duplicated. The data and the quantification were rechecked and the mistake was found only in Figure $2 E$. The correct Figure 2 is shown below. This correction does not affect interpretation or conclusions of the study.

A
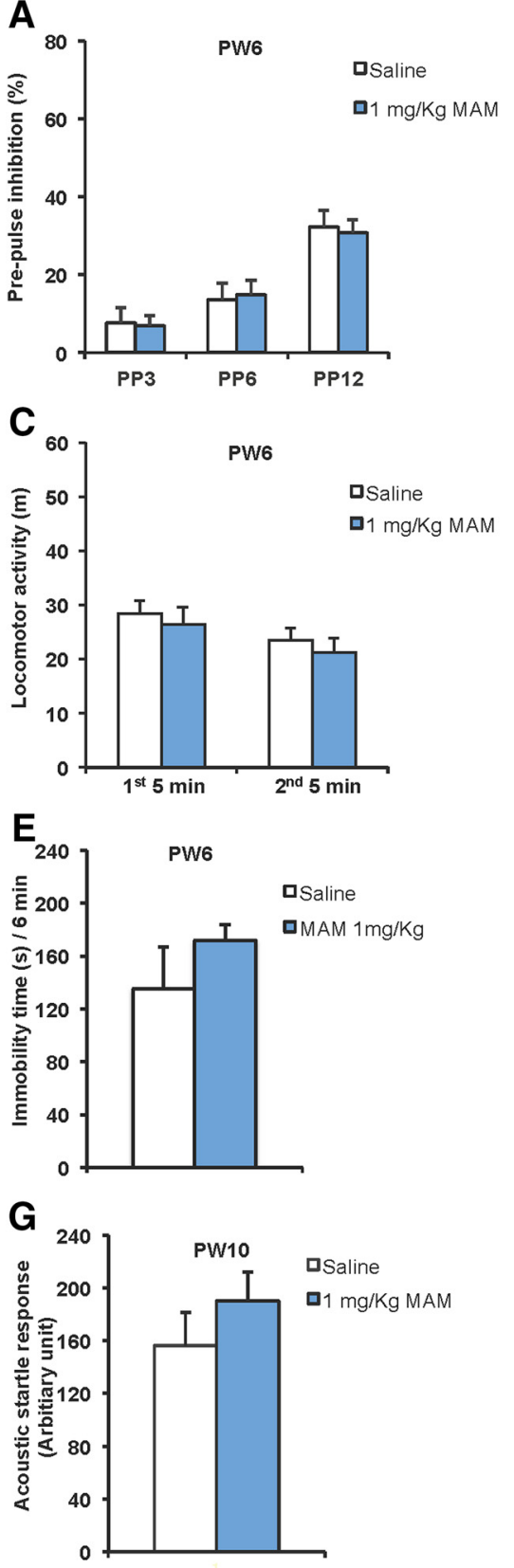

B

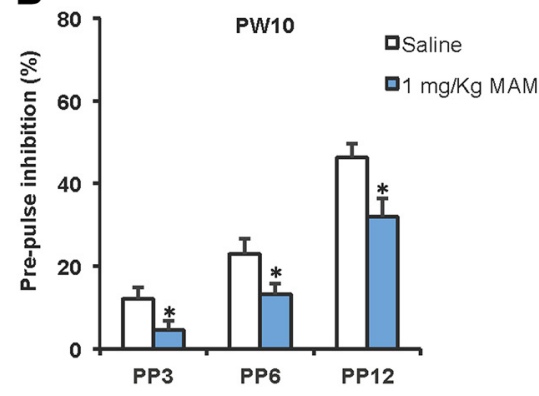

D

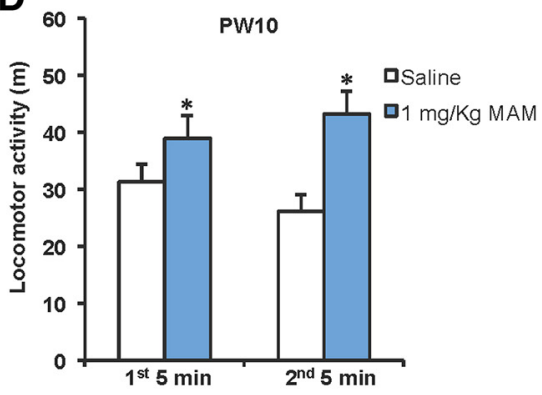

$\mathbf{F}$

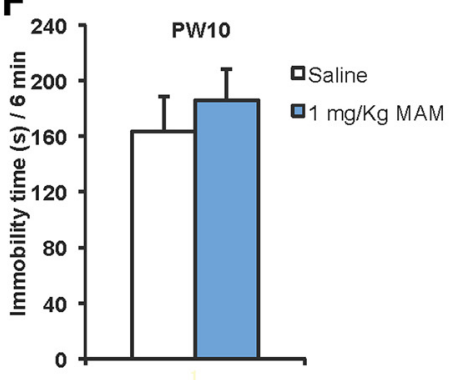

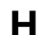

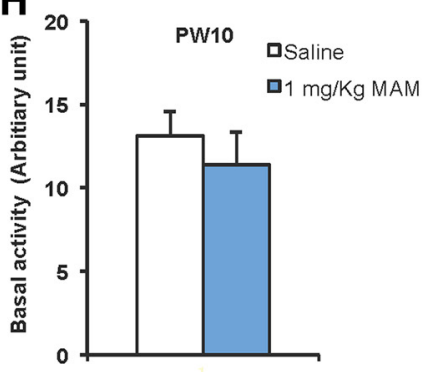

Figure 2. 